\title{
Efecto de la modificación de la tubería de salida en filtros de arena con brazos colectores
}

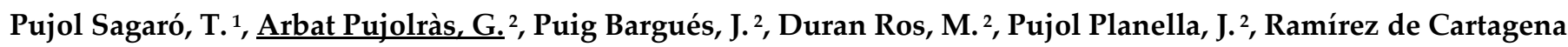 \\ Bisbe, F. ${ }^{2}$
}

Departamento de Ingeniería Mecánica y de la Construcción Industrial, Universidad de Girona, toni.pujol@udg.edu

Departamento de Ingeniería Química, Agraria y Tecnología Agroalimentaria, gerard.arbat@udg.edu, jaume.puig@udg.edu, miquel.duranros@udg.edu, joan.pujol@udg.edu, francisco.ramirez@udg.edu

Resumen: Los filtros presurizados de arena utilizados para proteger de las obturaciones las instalaciones de riego por goteo se pueden dividir en tres zonas en función de su comportamiento hidráulico: 1) entrada del agua, 2) lecho de arena, y 3) salida del agua. Estudios anteriores realizados para optimizar el funcionamiento hidráulico de este tipo de filtros se centraron en la zona de entrada y en el lecho de arena, proponiendo distintos cambios en el diseño tanto del difusor en la zona de entrada como del drenaje del lecho de arena. En el presente trabajo se analizan las consecuencias de modificar la zona de salida del agua de un filtro comercial de arena con brazos colectores sin variar su sistema de drenaje. El sistema original de salida del agua consistía en una tubería horizontal conectada a 10 brazos horizontales. Cinco diseños alternativos que diferían en su área transversal (original o doble), orientación (horizontal o vertical) y número (simple o doble) fueron numéricamente estudiados mediante dinámica de fluidos computacional en cuatro condiciones distintas de operación (dos alturas de lecho (160 y 260 $\mathrm{mm}$ ) y dos caudales (6 y $\left.12 \mathrm{~m}^{3} \mathrm{~h}^{-1}\right)$ ). La comparación de estos diseños con el comercial indicó que: 1) una tubería de salida con el doble del área transversal redujo la caída de presión total del filtro un $12 \%$ e incrementó la uniformidad del flujo del agua entre brazos colectores; 2) una doble tubería de salida conllevó un comportamiento simétrico del caudal respecto el brazo central pero no impidió que por el brazo central continuara circulando menor cantidad de agua que por los otros brazos colectores; y 3) una salida de agua vertical de igual diámetro que la comercial causó una caída de presión similar pero, en cambio, aumentó considerablemente la uniformidad del flujo entre los distintos brazos.

Palabras clave: filtración; filtro granular, riego por goteo, dinámica de fluidos computacional, modelización. 


\title{
Effect of the modification of the outlet pipe in sand filters with collector arms
}

\author{
Pujol Sagaró, T. ${ }^{1}$, Arbat Pujolràs, G. ${ }^{2}$, Puig Bargués, J. ${ }^{2}$, Duran Ros, M. ${ }^{2}$, Pujol Planella, J. ${ }^{2}$, Ramírez de Cartagena \\ Bisbe, F. ${ }^{2}$ \\ Departamento de Ingeniería Mecánica y de la Construcción Industrial, Universidad de Girona, toni.pujol@udg.edu \\ Departamento de Ingeniería Química, Agraria y Tecnología Agroalimentaria, gerard.arbat@udg.edu, jaume.puig@udg.edu, miquel.du- \\ ranros@udg.edu, joan.pujol@udg.edu, francisco.ramirez@udg.edu
}

\begin{abstract}
Pressurized sand filters that protect drip irrigation systems from clogging can be divided into three zones based on their hydraulic behaviour: 1) water inlet, 2) sand bed, and 3) water outlet. Previous studies carried out to optimize the hydraulic performance of this type of filters focused on the inlet zone and the sand bed, proposing different changes in the design of both the inlet diffuser and the drainage of the sand media. In this work, the consequences of modifying the water outlet area of a commercial sand filter with collector arms without changing its drainage system are analysed. The original water outlet system consisted of a horizontal pipe connected to 10 horizontal arms. Five alternative designs that differed in their cross-sectional area (original or double), orientation (horizontal or vertical), and number (single or double) were numerically studied using computational fluid dynamics under four different operating conditions (two bed heights (160 and $260 \mathrm{~mm}$ ) and two flow rates (6 and $\left.12 \mathrm{~m}^{3} \mathrm{~h}^{-1}\right)$ ). Comparison of these designs with the commercial one indicated that: 1) an outlet pipe with twice the cross-sectional area reduced the total pressure drop of the filter by $12 \%$ and increased the uniformity of the water flow between the collector arms; 2) a double outlet pipe entailed a symmetrical behaviour of the flow with respect to the central arm but did not prevent that less water would continue to circulate through the central arm than through the other collecting arms; and 3) a vertical water outlet of the same diameter as the commercial one caused a similar pressure drop but, on the other hand, considerably increased the uniformity of the flow between the different arms.
\end{abstract}

Keywords: filtration; granular filter, drip irrigation, computational fluid dynamics, modelling. 


\section{Introducción}

Recientemente, se ha prestado mucha atención al diseño de los elementos auxiliares internos y su efecto sobre la caída de presión en los filtros de arena utilizados en las instalaciones de riego localizado. El efecto del drenaje, que es el elemento situado en la base de arena cuyas ranuras, de menor anchura que el tamaño del medio filtrante, permiten la salida del agua filtrada ha sido estudiado por varios autores. Arbat et al. [1] demostraron la relevancia de los elementos de drenaje mediante un modelo numérico aplicado a un filtro comercial. Observaron que el flujo de agua se aceleraba dentro de la arena a medida que se acercaba a las ranuras del drenaje. Este aumento de velocidad dentro del medio poroso aumentó la caída de presión a valores mucho más altos que los esperados de la ecuación de Ergun aplicada al flujo uniforme.

Los rediseños de los elementos de drenaje de filtros comerciales también han investigados, tanto experimental como numéricamente. Bové et al. [2] llevaron a cabo un estudio de Dinámica de Fluidos Computacional (CFD) proponiendo un rediseño de un drenaje de crepinas comercial capaz de reducir la caída de presión total del filtro hasta en un 30\% para una velocidad superficial de alrededor $72 \mathrm{~m} \mathrm{~h}$ 1 . Bové et al. [3] posteriormente propusieron un tipo completamente nuevo de sistema de drenaje que maximizaba la superficie disponible conectada con la cámara de salida, ya que ocupaba toda el área de la sección transversal del filtro. Los estudios numéricos indicaron que este diseño desarrolló un flujo de agua más uniforme dentro de la arena, minimizando así las pérdidas de carga en el medio.

Mesquita et al. [4] se centraron en el diseño del difusor, por su papel en la uniformidad del flujo por encima de la región del lecho de arena. Sin embargo, el diseño de la región de salida también puede tener implicaciones importantes en el rendimiento hidráulico del filtro puesto que las características del colector de salida determinan la caída de presión y afectan a la distribución del agua a cada una de las unidades de drenaje.

El objetivo del presente trabajo fue analizar el efecto de distintos diseños de la tubería de salida en términos de 1) caída de presión total del filtro y 2) uniformidad de flujo dentro del medio poroso.

\section{Materiales y métodos}

\subsection{Diseño de la tubería de salida}

Todos los tipos de tubería de salida analizados fueron diseñados para el filtro de arena comercial FA1M del fabricante LAMA [5].

El agua filtrada se drenaba a través de 10 drenes de tipo brazo horizontal, los cuales se distribuyeron simétricamente con respecto al plano vertical central (Fig. 1). Cada brazo contenía 24 ranuras rectangulares de $79 \mathrm{~mm}$ de largo y $0,5 \mathrm{~mm}$ de ancho. Por lo tanto, el área abierta total por tubo fue igual a $960 \mathrm{~mm}^{2}$, lo que dio un área abierta total del sistema de drenaje inferior igual a $9600 \mathrm{~mm}^{2}$. En la configuración comercial, todos los brazos estaban conectados a una tubería de salida horizontal de diámetro interior $\mathrm{Do}=40,8 \mathrm{~mm}$.

Se propusieron cinco variaciones del diseño de la tubería de salida comercial, cuyas principales características se indican en la Tabla 1 (Fig. 2). Los diseños H2a y V2a tenían áreas de sección transversal el doble que las originales. El diseño H2d tenía un diámetro de sección transversal dos veces mayor que el original, por lo que tenía un área de sección transversal cuatro veces más grande que la original y dos veces mayor que la de los diseños H2a y V2a. Finalmente, se analizó el efecto de usar una salida gemela (diseño $2 \mathrm{H} ;$ Fig. 2). La longitud total del sistema de tuberías utilizado en las versiones $\mathrm{H}, \mathrm{H} 2 \mathrm{a}$ y H2d fue de $690 \mathrm{~mm}$, mientras que en las configuraciones V y V2a fue de $650 \mathrm{~mm}$, con la tubería de 
salida vertical centrada de $350 \mathrm{~mm}$ de largo. El sistema de tuberías tenía una longitud mucho mayor que la original en la caja de salida doble $2 \mathrm{H}$ (1080 $\mathrm{mm}$ de longitud total).
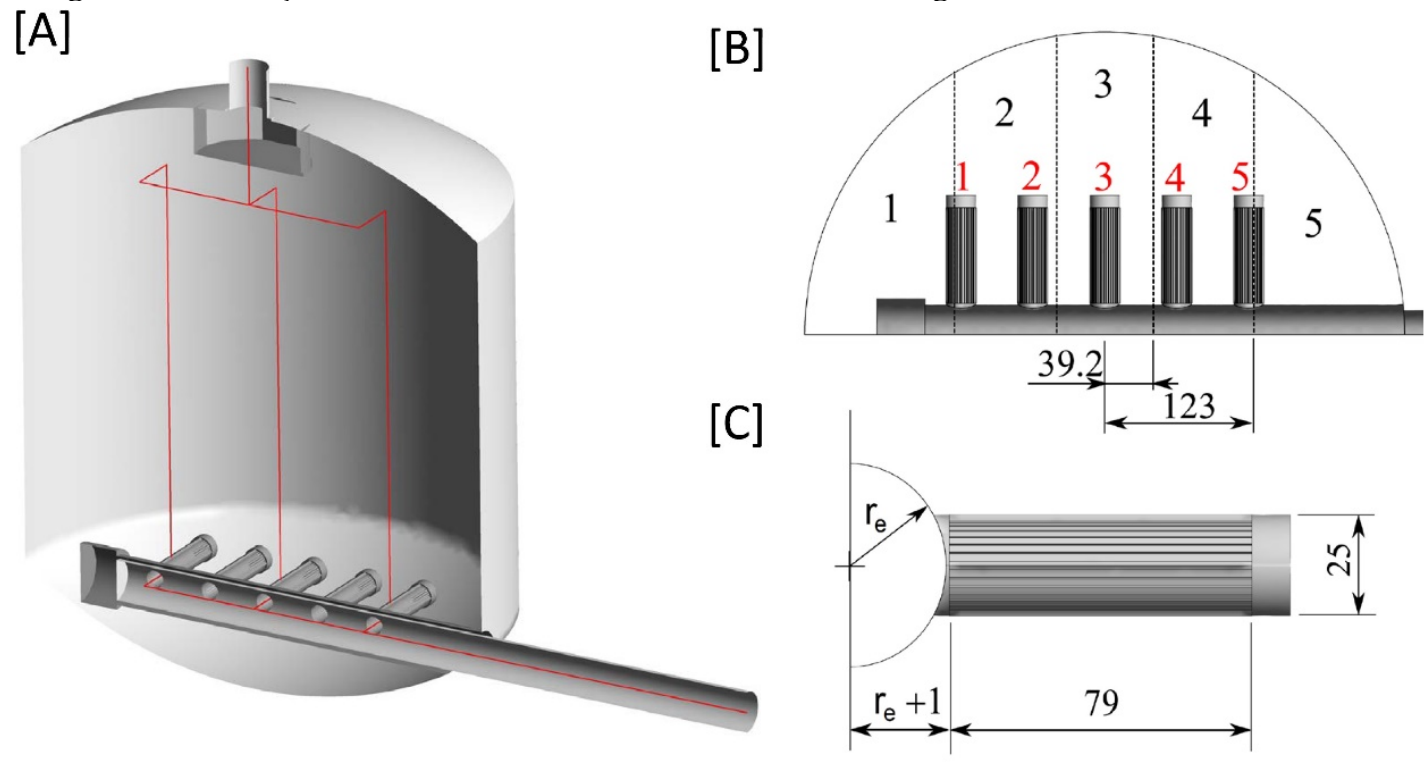

Fig. 1 - Vista de media sección del filtro comercial (caso H en la Tabla 1) donde las tres líneas rojas indican los perfiles analizados en la Sección 3 [A]. Media vista superior del colector de salida con la numeración de brazos (en rojo) y regiones de igual superficie (en negro) [B]. Dimensiones del tubo principal con $r_{e}$ el radio externo del tubo colector [C]. Dimensiones en mm.

Tabla 1. Características geométricas de los diseños de tubería de salida analizados.

\begin{tabular}{cccc}
\hline Diseño & Orientación & Diámetro interior & Área de salida \\
& & $(\mathrm{mm})$ & $\left(\mathrm{mm}^{2}\right)$ \\
\hline$H$ & Horizontal & 40,8 & 1307,4 \\
$H 2 a$ & Horizontal & 57,7 & 2614,8 \\
$H 2 d$ & Horizontal & 81,6 & 5229,6 \\
$V$ & Vertical & 40,8 & 1307,4 \\
$V 2 a$ & Vertical & 57,7 & 2614,8 \\
$2 H$ & Horizontal (2 salidas) & $2 \times 40,8$ & $2 \times 1307,4$ \\
\hline
\end{tabular}




\section{Congreso Nacional de Riegos CARTAGENA 2021}

[B] $\mathrm{H} 2 \mathrm{a}$

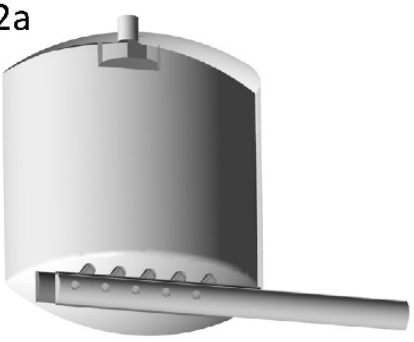

[C] $\mathrm{H} 2 \mathrm{~d}$

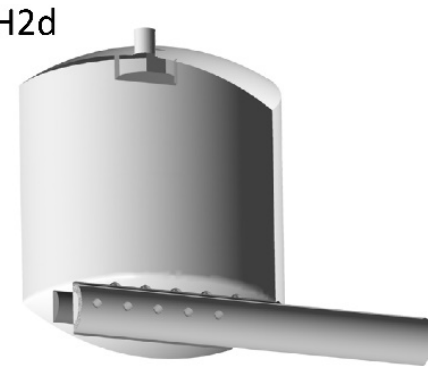

[D]

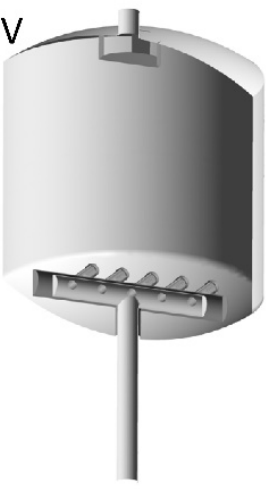

[E] V2a

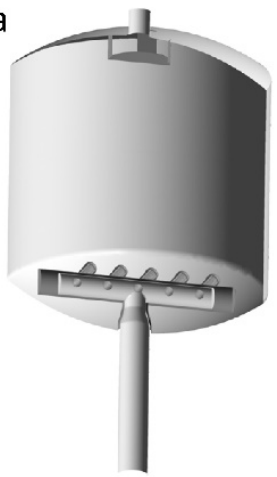

[F] $2 \mathrm{H}$

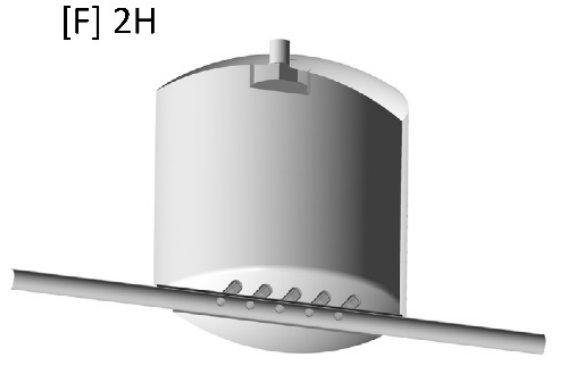

Fig. 2 - Diseños de tubería de salida analizados: caso comercial original (diámetro interior de salida Do) [A], caso H2a (D = $\sqrt{2}$ Do) [B], caso H2d $(\mathrm{D}=2 \mathrm{Do})[\mathrm{C}]$, caso $\mathrm{V}(\mathrm{D}=\mathrm{Do})[\mathrm{D}]$, caso V2a $(\mathrm{D}=\sqrt{ } 2 \mathrm{Do})[\mathrm{E}]$, caso $2 \mathrm{H}(\mathrm{D}=$ Do en ambas salidas) [F] (ver Fig. 1 y Tabla 1$)$.

\subsection{Modelo numérico}

El modelo numérico usado fue el código de dinámica de fluidos computacional (CFD) comercial ANSYS-Fluent, que utiliza el método de volumen finito para resolver las ecuaciones que gobiernan la dinámica de flujo (ANSYS, 2020). Varios autores han aplicado este software con éxito para simular el comportamiento de filtros de arena presurizados $[1,3,5,6]$. En todos los casos aquí considerados, el dominio de simulación correspondió a la mitad de todo el cuerpo ya que se aprovecharon las condiciones de simetría a lo largo del plano vertical central. Como en Pujol et al. [6], el medio filtro se dividió en tres zonas, siendo: 1) desde la entrada hasta la superficie del lecho de arena; 2) lecho filtrante; 3 ) desde la entrada del desagüe del lecho hasta la salida del colector. Las zonas 1 y 3 anteriores se definieron como regiones ocupadas solo por agua. La zona 2 se definió como un medio poroso homogéneo, por lo que se agregó un término de sumidero de impulso a la ecuación de Navier-Stokes. Este término sumidero S_i (Pa m-1) siguió de la ecuación de Ergun, estando compuesto por dos términos,

$$
S_{i}=-\frac{1}{\alpha} \mu v_{i}-C_{2} \frac{\rho}{2}|v| v_{i} \quad \text { for } i=x, y, z
$$

donde se usaron propiedades de fluido de agua constante para viscosidad $\mu(=0.001003 \mathrm{~Pa}$ s) y densidad $\left(\rho=998,2 \mathrm{~kg} \mathrm{~m}^{-3}\right), \alpha\left(\mathrm{m}^{2}\right)$ y $\mathrm{C}_{2}\left(\mathrm{~m}^{-1}\right)$ fueron la permeabilidad y el factor de resistencia inercial de la arena, respectivamente. En la ecuación (1), $v_{i}\left(\mathrm{~m} \mathrm{~s}^{-1}\right)$ fue el i-ésimo componente de la velocidad superficial del flujo cuya magnitud fue $|\mathrm{v}|$. La velocidad superficial se calculó como la velocidad sin tener en cuenta el medio poroso (es decir, sin porosidad). El flujo se consideró laminar dentro de la zona de arena. 
Todos los diseños se simularon en cuatro condiciones operativas diferentes, siendo la combinación de dos alturas de lecho de arena $\mathrm{H}_{\mathrm{s}}(160 \mathrm{~mm}$ y $260 \mathrm{~mm})$ y dos caudales volumétricos $\mathrm{Q}\left(6 \mathrm{~m}^{3} \mathrm{~h}^{-1} \mathrm{y} 12 \mathrm{~m}^{3} \mathrm{~h}^{-}\right.$ $\left.{ }^{1}\right)$. Estos valores de caudal correspondieron a velocidades superficiales del filtro iguales a $30,6 \mathrm{~m} \mathrm{~h}^{-1} \mathrm{y}$ $61,1 \mathrm{~m} \mathrm{~h}^{-1}$, respectivamente.

La configuración de los parámetros de la malla fue igual a la empleada en Pujol et al. [5,6]. Los elementos utilizados para discretizar todos los cuerpos fueron tetraedros excepto en las regiones adheridas a las paredes internas del filtro en las que se aplicó una capa de cinco prismas. El tamaño máximo de los elementos fue de $0,3 \mathrm{~mm}$ en las ranuras del desagüe, $0,8 \mathrm{~mm}$ en las paredes del desagüe, y $3 \mathrm{~mm}$ en la placa difusora, tubo de entrada y tubo de salida. En los dominios de volumen, los elementos tenían un tamaño máximo de $3 \mathrm{~mm}$ dentro de los elementos de drenaje inferior y $10 \mathrm{~mm}$ dentro del dominio del filtro. El factor de crecimiento fue del $20 \%$. Con esta configuración, el número total de elementos necesarios para mallar la mitad del filtro para el caso $\mathrm{H}$ en la Tabla 1 fue de 8,9 × 106 elementos.

\section{Resultados y discusión}

\subsection{Diseño de la tubería de salida}

La caída de presión del filtro $\Delta p_{f}$ para las cuatro condiciones de trabajo y los seis diseños de tubería de salida de las Figs. 1-2 se muestran en la Fig. 3. Para todos los casos, el valor máximo correspondió a la mayor altura del lecho de arena $(260 \mathrm{~mm})$ y caudal $\left(=12 \mathrm{~m}^{3} \mathrm{~h}^{-1}\right)$. La variación media de la caída de presión del filtro entre las configuraciones $H s=160 \mathrm{~mm}$ y $\mathrm{Hs}=260 \mathrm{~mm}$ fue $4757 \mathrm{~Pa}$ y $9525 \mathrm{~Pa}$ para caudales $\mathrm{Q}=6 \mathrm{~m}^{3} \mathrm{~h}^{-1}$ y $\mathrm{Q}=12 \mathrm{~m}^{3} \mathrm{~h}^{-1}$, respectivamente. Debe considerarse que estos valores corresponden casi exactamente a la caída de presión predicha por la ecuación (1) para una columna de arena de $260-160=100 \mathrm{~mm}$ de altura en ambos modos de velocidad superficial analizados. Esto significó que el flujo dentro de la arena fue muy uniforme, al menos a lo largo de los primeros $100 \mathrm{~mm}$. La caída de presión del filtro para los casos $H 2 a, H 2 d$ y $2 H$ fue 12,4\%, 15,6\% y 13,0\% inferior a la de la configuración original, caso H. Sin embargo, estos valores se redujeron para tener en cuenta que la tubería de salida se conectaría a una tubería existente en la instalación hidráulica de un solo tubo de diámetro interior Do. Por lo tanto, los valores de $\Delta p f$ en la Fig. 3 se modificaron restando las pérdidas de carga secundarias mínimas requeridas para conectarse al sistema de tuberías externo. Los valores del coeficiente de pérdidas secundarias se extrajeron de Çengel y Cimbala [7]. Una vez que se tuvo en cuenta esta pequeña contribución a la pérdida, la variación de la caída de presión del filtro $\Delta p f$ con respecto al valor del diseño original ( $\Delta p f, H$ en la Fig.3) reveló que los diseños $H 2 a$ y $H 2 d$ proporcionaron mejoras sustanciales (reducciones superiores al 10\%, Fig. 4). Sin embargo, el diseño $2 \mathrm{H}$ fue claramente penalizado debido a las pérdidas secundarias. La configuración del colector vertical propuesta en la Fig. 2 casi produjo un valor de $\Delta p f$ idéntico al del filtro original. 


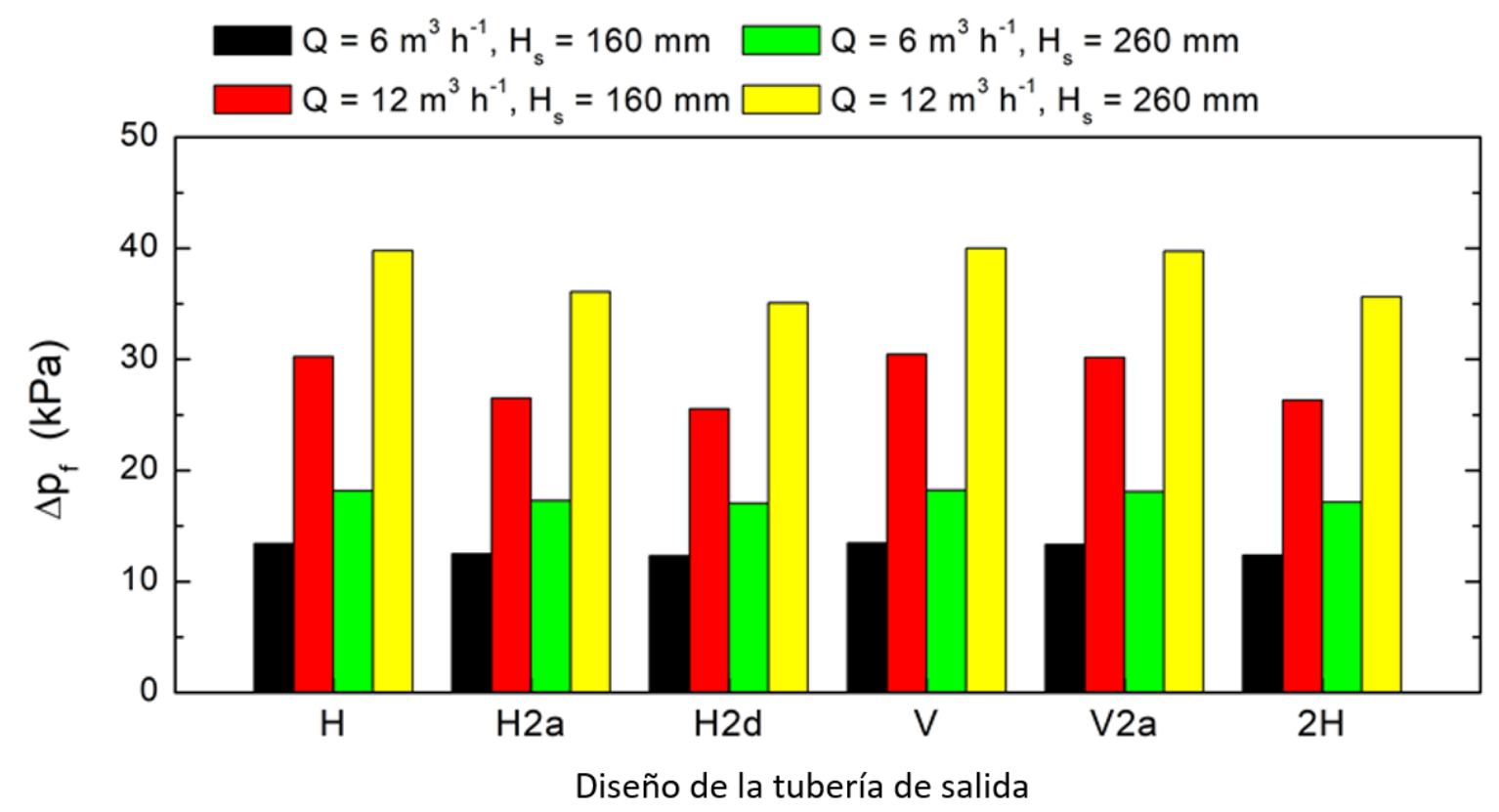

Fig. 3 - Caída de presión del filtro para filtros con diferentes diseños de tubería de salida (Tabla 1) en diferentes modos de trabajo (caudal Q y altura del lecho de arena Hs).

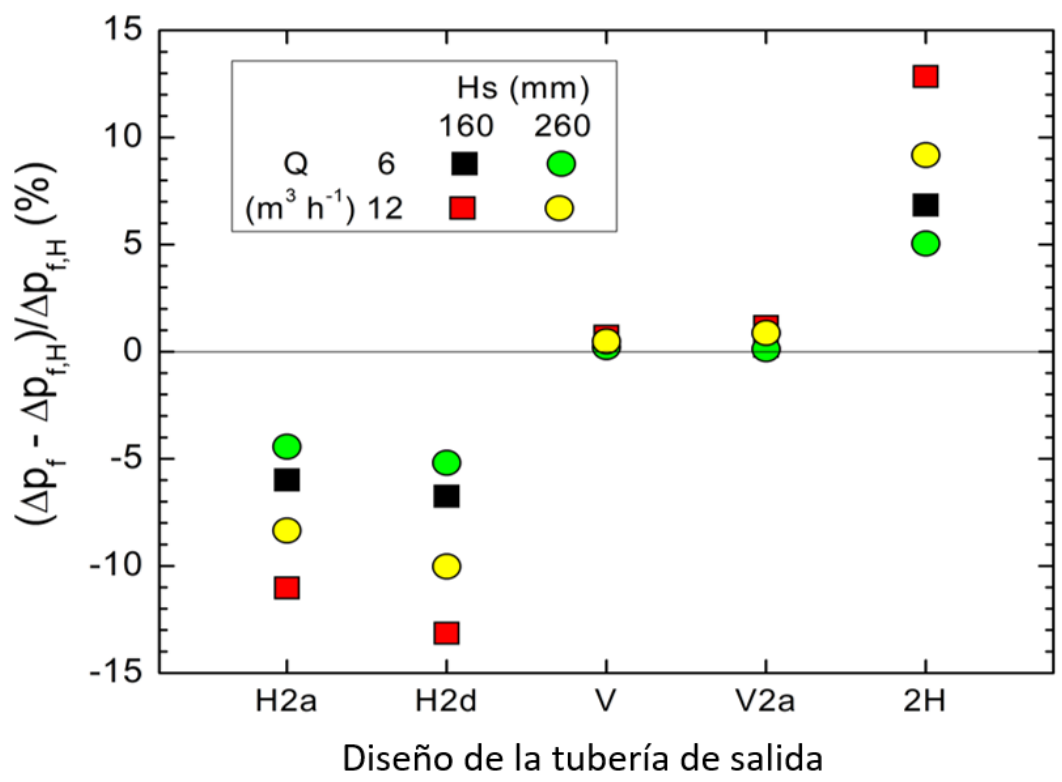

Fig. 4 - Variación (\%) de la caída de presión del filtro con respecto al filtro original con diferentes diseños de tubería de salida, incluyendo las pérdidas secundarias para adaptarse a una tubería externa de diámetro Do en diferentes modos de trabajo (caudal Q y altura del lecho de arena $\mathrm{Hs}$ ).

Cada una de las tres zonas (entrada de agua, arena, salida de agua) en las que se puede dividir el filtro contribuyó de manera diferente a las pérdidas de presión totales (Fig. 5). En aras de la concreción, los siguientes resultados se refieren al caso $\mathrm{Hs}=160 \mathrm{~mm}, \mathrm{Q}=12 \mathrm{~m}^{3} \mathrm{~h}^{-1}$, aunque las conclusiones extraídas fueron válidas para otros modos de funcionamiento. Como era de esperar, la columna de arena tuvo el aporte principal con un comportamiento que claramente se apartó del uniforme a medida que el flujo 


\section{Congreso Nacional de Riegos CARTAGENA 2021}

se acercaba a los drenes de brazo. En la Fig. 5, por conveniencia, se divide la región de arena en dos partes. La primera correspondió a los primeros $100 \mathrm{~mm}$ (desde la parte superior de la superficie del lecho de arena, $160 \mathrm{~mm}$, hasta $60 \mathrm{~mm}$ por encima del nivel de los brazos). La segunda comprendía la capa de arena desde $60 \mathrm{~mm}$ por encima de la altura de los drenes hasta el nivel de los brazos. La última región, aunque solo tenía $60 \mathrm{~mm}$ de altura, tuvo el mayor efecto sobre la pérdida de presión general (Fig. 5). Este efecto se debió al aumento en la velocidad del flujo dentro del lecho de arena, a medida que las líneas de corriente convergían hacia las ranuras del drenaje subterráneo (alta velocidad en la ecuación (1)), y también porque la trayectoria real del agua dentro de la arena no fue recta, sino que se curvó para alcanzar las aberturas del brazo de drenaje. Esto contrastó con la trayectoria casi totalmente recta de la primera región (de $160 \mathrm{~mm}$ a $60 \mathrm{~mm}$ ).

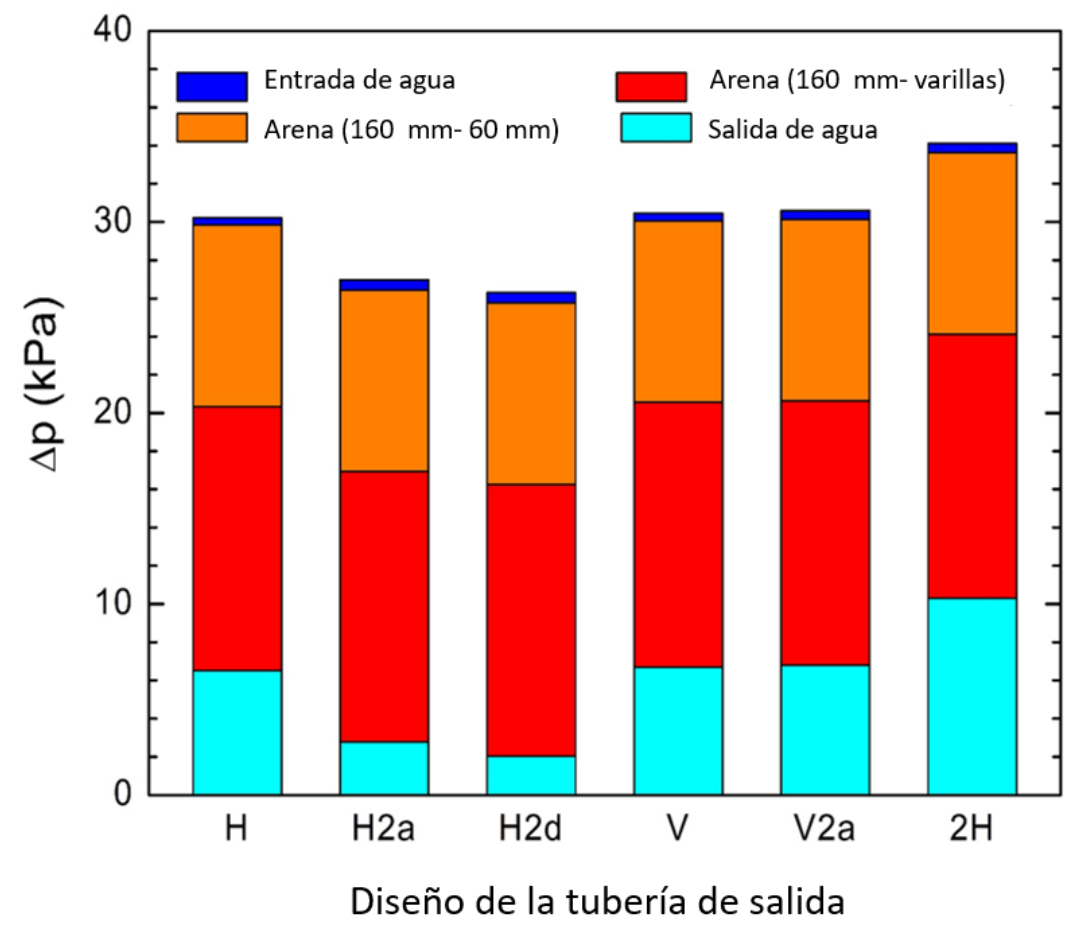

Fig. 5 - Contribución de diferentes zonas a la caída de presión total del filtro para diferentes diseños de tubería de salida, incluidas pérdidas secundarias para adaptarse a una tubería externa de diámetro Do correspondiente a Q = $12 \mathrm{~m}^{3} \mathrm{~h}^{-1}$ y Hs $=160 \mathrm{~mm}$.

La zona que menos contribuyó a la caída total de presión del filtro fue la región de entrada de agua (Fig. 5).

La zona de salida de agua, incluidos los accesorios necesarios para terminar con el mismo diámetro interior de la tubería que la comercial (caso H) tuvo una contribución notable en la caída de presión total. Para el modo de operación $\mathrm{Q}=12 \mathrm{~m}^{3} \mathrm{~h}^{-1} \mathrm{y} \mathrm{Hs}=160 \mathrm{~mm}$ fue $21 \%$ de $\Delta p f$ para el caso de referencia $(\mathrm{H})$ pero se redujo a solo $10 \%$ y $8 \%$ para diseños $\mathrm{H} 2$ a y $\mathrm{H} 2 \mathrm{~d}$, respectivamente. Las mejoras para las otras configuraciones estudiadas fueron más modestas pero aún notorias.

La reducción de las pérdidas de carga en los diseños propuestos, además de disminuir la demanda de energía, también supuso aumentar la uniformidad del flujo dentro de la arena. Esta mayor uniformidad en el flujo resultó de suma importancia para evitar la formación de caminos preferenciales dentro de la arena que acelerarían la obstrucción del filtro o afectarían negativamente el rendimiento del filtro. Por lo tanto, se investigó cómo se distribuyó el caudal total a lo largo del área de la sección transversal del 


\section{Congreso Nacional de Riegos CARTAGENA 2021}

filtro dentro de la región de arena a diferentes alturas. Las secciones horizontales se dividieron en cinco superficies de igual área (Fig. 1B) en las que se calculó el caudal (Fig. 6). La ubicación a una altura de $158 \mathrm{~mm}$ estaba a solo $2 \mathrm{~mm}$ por debajo de la capa del lecho de arena. En esa posición, el patrón fue similar para todos los diseños de tubería de salida, con la región \# 5 (la más cercana a la salida en la configuración horizontal de tubería única) teniendo la mayor disparidad de flujo (Fig. 6A). A $60 \mathrm{~mm}$ por encima del nivel del desagüe (Fig. 6B), la variación de caudal entre las tres regiones centrales y las laterales aumentó. Además, hubo una clara asimetría para el caso $\mathrm{H}$ con respecto al plano del brazo central, con las regiones \# 4 y \# 5 participando más en el flujo de agua que sus contrapartes \# 2 y \# 1 . En cambio, los diseños con doble salida y salida vertical central tuvieron un comportamiento simétrico a cualquier altura dentro del medio poroso.

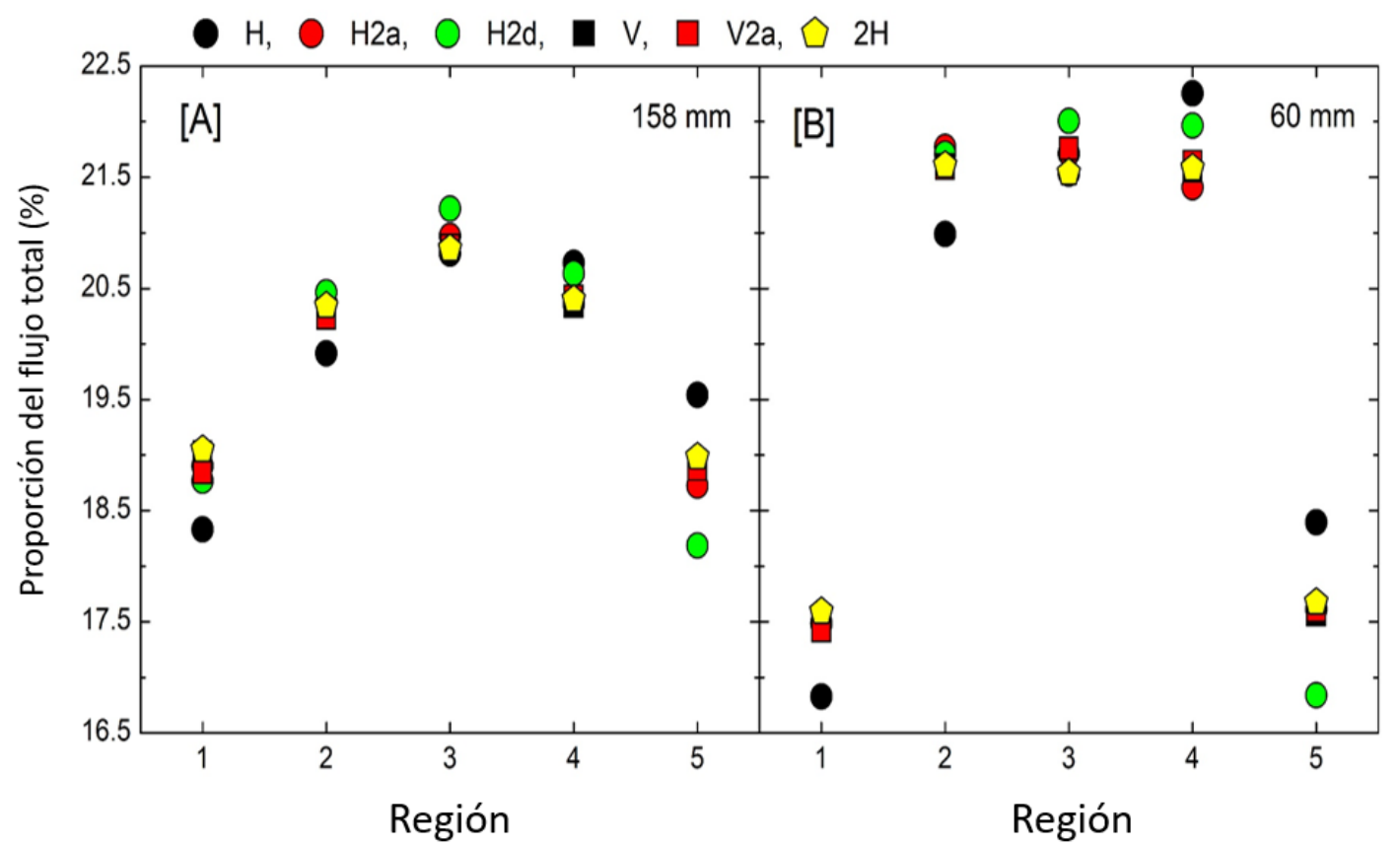

Fig. 6 - Proporción de flujo (en \% del valor total) que cruzó superficies de igual área (ver regiones en la Fig. 1B) en planos horizontales ubicados a $158 \mathrm{~mm}$ [A] y $60 \mathrm{~mm}$ [B] por encima del nivel del drenaje. Caso Q $=12 \mathrm{~m}^{3} \mathrm{~h}^{-1} \mathrm{y} \mathrm{Hs}=160 \mathrm{~mm}$.

\section{Conclusiones}

Se ha analizado numéricamente el efecto del diseño de la tubería de salida en el rendimiento hidráulico de un filtro de arena presurizado con 10 drenaje formado por brazos. Se investigaron cinco diseños que variaban en diámetro, orientación y número de salidas y se compararon los resultados con los del filtro comercial original en cuatro modos de funcionamiento. Las simulaciones indicaron que la configuración original de salida horizontal única produjo un desequilibrio en el caudal drenado por cada drenaje ( $42 \%$ de variación). Esto puede favorecer la creación de caminos preferentes dentro de la columna de arena, como se sugiere después de analizar el flujo de agua y las diferencias de presión entre diferentes sectores dentro del medio poroso.

Doblar el área de la sección transversal de la tubería de salida redujo la caída de presión del filtro hasta un $12 \%$, mejorando también el equilibrio hidráulico entre los drenajes inferiores ( $28 \%$ de variación). Un aumento adicional del área de la sección transversal de la tubería de salida (4 veces la original) solo pudo reducir la caída de presión en un 3\% con respecto duplicar el área de la sección transversal original. Además, no proporcionó una mejora relevante en el balance hidráulico de los drenajes (se 


\section{Congreso Nacional de Riegos CARTAGENA 2021}

observaron variaciones del 25\%). El cambio de la orientación de la tubería de salida a una vertical hacia abajo en la posición central no redujo las pérdidas totales de energía del filtro, pero mejoró sustancialmente la uniformidad del flujo en cada drenaje ( $21 \%$ de variación entre elementos, siendo dos veces menor que la variación del diseño comercial). Un aumento en el diámetro de la tubería de salida no modificó sustancialmente las cifras anteriores. Finalmente, una salida gemela proporcionó un comportamiento de flujo simétrico perfecto con respecto al tubo central pero no aumentó el equilibrio en comparación con el diseño de salida vertical hacia abajo, mantuvo la tubería central con menos volumen de agua drenada y, una vez incluidos todos los accesorios necesarios para conectarse a la instalación, aumentó la caída de presión general del filtro hasta un $13 \%$.

A partir de los resultados anteriores, sugerimos una revisión del diseño de la tubería de salida horizontal comercial, ya sea aumentando su diámetro al doble del original o modificando su orientación a centrada y hacia abajo. Ambos son sencillos de implementar y tienen efectos hidráulicos similares, aunque se preferiría el vertical debido a mayor efecto sobre el comportamiento simétrico.

\section{Agradecimientos}

Los autores agradecen a la Agencia Española de Investigación y al Fondo Europeo de Desarrollo Regional por su apoyo económico a través del proyecto RTI2018-094798-B-100.

\section{Referencias}

1. Arbat, G.; Pujol, T.; Montoro, L. Using computational fluid dynamics to predict head losses in the auxiliary elements of a microirrigation sand filter. Trans. ASABE 2011, 54, 1367-1376.

2. Bové, J.; Arbat, G.; Pujol, T.; Duran-Ros, M.; Ramírez de Cartagena, F.; Velayos, J.; Puig-Bargués, J. Reducing energy requirements for sand filtration in microirrigation: Improving the underdrain and packing. Biosyst. Eng. 2015, 140, 67-78, doi:10.1016/j.biosystemseng.2015.09.008.

3. Bové, J.; Puig-Bargués, J.; Arbat, G.; Duran-Ros, M.; Pujol, T.; Pujol, J.; Cartagena, F.R. de Development of a new underdrain for improving the efficiency of microirrigation sand media filters. Agric. Water Manag. 2017, doi:10.1016/j.agwat.2016.06.031.

4. $\quad$ Mesquita, M.; de Deus, F.P.; Testezlaf, R.; da Rosa, L.M.; Diotto, A. V. Design and hydrodynamic performance testing of a new pressure sand filter diffuser plate using numerical simulation. Biosyst. Eng. 2019, 183, 58-69, doi:10.1016/j.biosystemseng.2019.04.015.

5. Pujol, T.; Puig-Bargués, J.; Arbat, G.; Vegas, A.; Duran-Ros, M.; Pujol, J.; Ramírez de Cartagena, F. Numerical study of the effects of pod, wand and spike type underdrain systems in pressurised sand filters. Biosyst. Eng. 2020, 200, 338-352, doi:10.1016/j.biosystemseng.2020.10.018.

6. Pujol, T.; Puig-Bargués, J.; Arbat, G.; Duran-Ros, M.; Solé-Torres, C.; Pujol, J.; Ramírez de Cartagena, F. Effect of wand-type underdrains on the hydraulic performance of pressurised sand media filters. Biosyst. Eng. 2020, 192, 176-187, doi:10.1016/j.biosystemseng.2020.01.015.

7. Çengel, Y..; Cimbala, J.M. Fluid mechanics: fundamentals and applications. In; McGraw-Hill: New York, 2017. 